\title{
Novel directory service and message delivery mechanism enabling scalable mobile agent communication
}

\author{
Jinho Ahn* \\ Department of Computer Science, Kyonggi University, Suwon, Korea
}

\begin{abstract}
Mobile agent technology has emerged as a promising programming paradigm for developing highly dynamic and large-scale service-oriented computing middlewares due to its desirable features. For this purpose, first of all, scalable location-transparent agent communication issue should be addressed in mobile agent systems despite agent mobility. Although there were proposed several directory service and message delivery mechanisms, their disadvantages force them not to be appropriate to both low-overhead location management and fast delivery of messages to agents migrating frequently. To mitigate their limitations, this paper presents a scalable distributed directory service and message delivery mechanism. The proposed mechanism enables each mobile agent to autonomously leave tails of forwarding pointers on some few of its visiting nodes depending on its preferences. This feature results in low message forwarding overhead and low storage and maintenance cost of increasing chains of pointers per host. Also, keeping mobile agent location information in the effective binding cache of each sending agent, the sending agent can communicate with mobile agents much faster compared with the existing ones.
\end{abstract}

Keywords: Distributed system, mobile agent, directory service, message delivery, scalability

\section{Introduction}

Thanks to its desirable features such as asynchronous and dynamic execution and autonomy $[12,17$, $22,25]$, mobile agent technology has emerged as a promising programming paradigm for developing highly dynamic and large-scale service-oriented computing middlewares in various fields like ubiquitous computing [1,3,5,9,10,26], web service [28], grid computing [13,15] and adhoc network [19]. However, as mobile agent systems become built on large-scale networks and the number of mobile agents is rapidly increasing, several research issues related to mobile agents should be reconsidered to satisfy this demand. Among them, it is most important to enhance the performance of the agent communication in large-scale infrastructures. For this purpose, some effective and efficient inter-agent communication mechanism is required in distributed agent-based systems. Agent mobility may lead to the loss of messages being destined to an agent on its migration. Thus, it causes reliable inter-agent communications to be not easy to achieve in the distributed agent based systems. Especially, guaranteeing the delivery of messages to highly mobile agents, which move frequently among service nodes, is a more challenging problem. In this paper, we attempt to address these issues effectively like in Fig. 1. Although there were proposed

\footnotetext{
*Address for correspondence: Department of C.S., Kyonggi University, San 94-6 Yiuidong, Yeongtonggu, Suwonsi Gyeonggido 443-760, Korea. Tel.: +82 31 249-9674; Fax: +82 31 249-9673; E-mail: jhahn@kyonggi.ac.kr.
} 


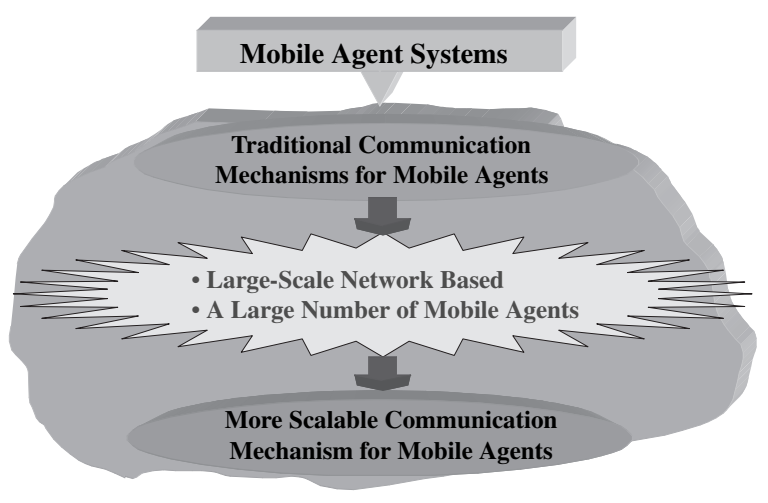

Fig. 1. The motivation of our work.

several directory service and message delivery mechanisms to address this issue as mentioned below, their disadvantages force them not to be appropriate to both low-overhead location management and fast delivery of messages to agents migrating frequently.

Firstly, the broadcast-based mechanism [18] guarantees transparent and reliable inter-agent communication and can also provide multicast communication to a set of agents. But, to locate the message destination, the mechanism has to contact every visiting node in the network. Thus, its large traffic overhead makes broadcasts impractical in large-scale distributed agent systems.

Secondly, in the home-based mechanism [14,21], every mobile agent has a home node and should register its current location with the home node whenever it moves. Thus, when some messages are sent to a mobile agent currently located at a foreign node, the messages are first directed to its home node, which forwards them to the agent. This mechanism is simple to implement and results in little mobile agent locating overhead. However, it is unsuitable for highly mobile agents in distributed agent based systems because every agent location updating and message delivery are all performed around the home node, which introduces centralization. Additionally, in the distributed agent-based systems, the home node may be disconnected from the network.

Lastly, the forwarding pointer-based mechanism $[11,16]$ forces each node on a mobile agent's movement path to keep a forwarding pointer to the next node on the path. Thus, if a message is delivered to an agent not being at the home node, the message must traverse a list of forwarding nodes. Thus, this mechanism can avoid performance bottlenecks of the global infrastructure, and therefore improve its scalability, particularly in large-scale distributed agent-based systems, compared with the home based one. Additionally, even if a home node is disconnected from the rest of the network, the forwarding pointer based mechanism allows agents registering with the node to communicate with other agents. However, as highly mobile agents leads to the length of their chains of pointers being rapidly increasing, its message forwarding overhead may be significantly larger. Furthermore, the number of forwarding pointers each service node needs to keep on its storage may exponentially increase if a large number of mobile agents are running in the systems. In a previous work [16], a type of update message called in form message was introduced to include an agent's current location for shortening the length of trails of forwarding pointers. In this case, a node that receives the message is allowed to update its table if the received information is more recent than the one it had. However, it introduces no concrete and efficient solutions for this purpose, for example, when update messages should be sent, and which node they should be sent to. In order to mitigate their limitations, this paper presents a scalable distributed directory service and message delivery mechanism. The proposed mechanism enables each mobile agent 
to autonomously leave tails of forwarding pointers on some few of its visiting nodes depending on its preferences. This feature results in low message forwarding overhead and low storage and maintenance cost of increasing chains of pointers per host. Also, keeping mobile agent location information in the effective binding cache of each sending agent, the sending agent can communicate with mobile agents much faster compared with the existing ones.

The rest of the paper is organized as follows. Section 2 describes our mobile agent system model assumed in this paper. In Section 3, we present our directory service and message delivery mechanism and prove its correctness. Section 4 makes several experiments for performance evaluation and Section 5 reviews related work. Finally, Section 6 concludes this paper.

\section{Mobile agent system model}

In this paper, we consider an asynchronous distributed agent based system where there is no global memory, no global clock and no bound on message delay. The system consists of a set of agent service nodes. Each service node supports an environment in which agents can operate safely and securely, and provides a uniform set of services that visiting agents can access its local resources in a limited way regardless of their locations. An agent is initially created on a service node, called home node of the agent, and is given a unique identifier within the node. So, each agent can be identified as a globally unique object in the system by using the combination of its local identifier and the identifier of its home node. When an agent migrates in the system, its code and state information are captured and then transferred to the next node. After arriving at the node, the mobile agent resumes and performs its task, if needed, by interacting with other agents. In order to perform an assigned task on behalf of a user, a mobile agent $m a$ executes on a sequence of $l(l>1)$ service nodes $I_{m a}=\left[N_{h o m e}, N_{1}, \cdots, N_{(l-1)}\right]$ according to its itinerary, which may be statically determined before the mobile agent is launched at its home node or dynamically while progressing its execution. It is assumed that communication channels support standard asynchronous message passing and are immune to partitioning, and reliable and FIFO. Mobile agents can migrate and messages be passed along these channels. Finally, we assume that each service node is failure-free because this makes it more easy to design and understand our agent tracking and message delivery mechanism explained hereafter.

\section{Scalable directory service and message delivery mechanism}

In this section, we first explain basic concept of our scalable mobile agent communication mechanism, describe its two components, directory service and message delivery algorithms in detail respectively, and then prove the correctness of the mechanism.

\subsection{Basic idea}

We attempt to develop our directory service and message delivery mechanism to have all three desirable features compared with the others as follows.

+ Alleviate the centralized dependency on the home node and its disconnection problem.

+ Require a small size of storage of each service node for agent location management.

+ Incur low message forwarding overhead. 


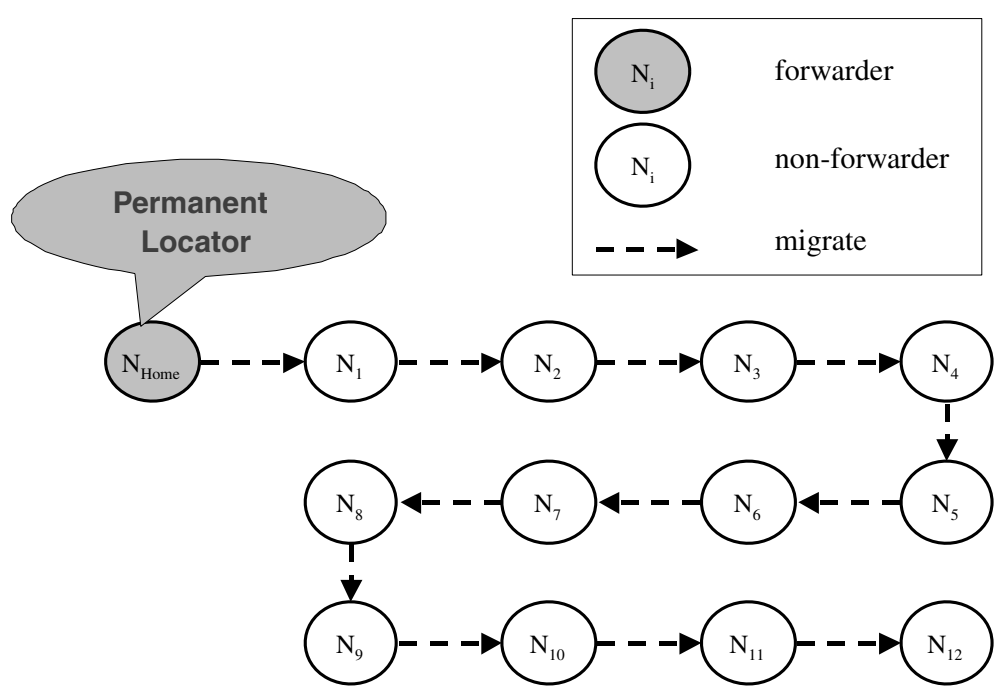

Fig. 2. Home-based mechanism.

Before discussing, the definition of two special directory service nodes should be explained. First, forwarder of an agent means a service node keeping a forwarding pointer of the agent on its storage. Thus, depending on the behavior of agent communication mechanisms, there may exist various number of forwarders of each agent in the system. Second, locator of an agent is the forwarder managing the identifier of the node where the agent is currently located. In this paper, it is assumed that there is only one locator in the system. We will explain how the proposed mechanism satisfies the three requirements using Figs 2, 3 and 4. These figures show all the same example that a mobile agent $m a$ performs its task while migrating from its home node to nodes $N_{1}$ through $N_{12}$ in node number order. In home-based mechanism, $N_{\text {home }}$ among all visiting nodes $\in I_{m a}$ is the only forwarder of agent $m a$ i.e., its permanent locator like in Fig. 2. Thus, this mechanism forces every agent created on a node to update its current location information only at the home node on every migration. Also, all messages destined to the agent must be always delivered to the agent only via its home node. Therefore, the mechanism doesn't address the first requirement.

In forwarding pointer-based mechanism, every visiting node $\in I_{m a}$ is a forwarder of agent $m a$ like in Fig. 3. Thus, when each agent moves to another node, the agent has only to register its location only with the right before visiting node. Additionally, each sent message can be transmitted to its target agent via the home node as well as other forwarders of the agent. Therefore, the mechanism can satisfy the first requirement. However, in this mechanism, whenever each agent, especially highly mobile agent, migrates to a new node, its previous node has to keep a forwarding pointer of the agent. This feature may lead to significantly increasing the size of storage on which each service node maintains location information of agents. Moreover, as the forwarding path for delivering each message to its target agent highly increases due to the feature like in Fig. 3, its delivery time also increases at the same rate. Therefore, neither the second requirement nor the third one is addressed in this mechanism.

To satisfy all the three requirements, our proposed mechanism enables each mobile agent to autonomously and systematically leave trails of forwarding pointers only on some few of its visiting nodes depending on its preferences such as location updating and message delivery costs, network topology, security policies, communication patterns and so on, where the methods for selecting suitable forwarders of each mobile agent based on its administrator's policy may be embedded in the software logic of the 


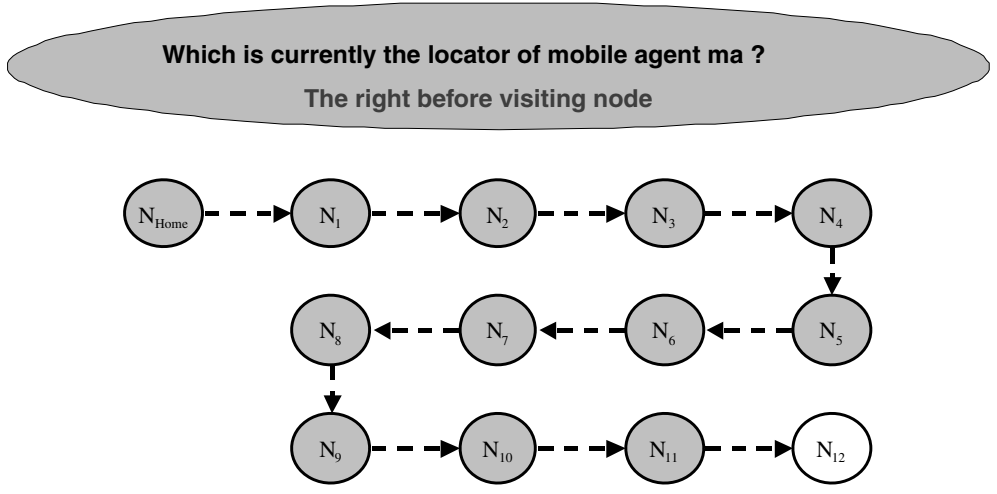

Fig. 3. Forwarding pointer-based mechanism.

agent. This behavior can considerably reduce both the amount of agent location information each node needs to maintain and the delivery time of each message because the length of its forwarding path may be much more shortened. Also, since there exist multiple forwarders, not only one, in this mechanism, the home node centralization can be avoided. But, as a part of the visiting nodes are forwarders in this mechanism, a new method is required to consistently manage location of each agent and enable each sent message to be delivered to the agent despite its migrations unlike in the previous forwarding pointer-based one. To satisfy the goal, the method is performed as follows. In Fig. 4, when agent $m a$ moves from $N_{\text {home }}$ to $N_{1}$, it forces $N_{1}$ to be its forwarder. In this case, $N_{1}$ becomes ma's locator. Afterwards in this method, $m a$ should register its location with $N_{1}$ on every migration until it moves to the next forwarder $N_{5}$. When the agent arrives at $N_{5}, N_{5}$ informs $N_{1}$ that $N_{5}$ plays a role of $m a$ 's locator from now on. Hereafter, $m a$ 's current location information is managed by $N_{5}$ until the agent arrives at its next forwarder $N_{10}$. This method may result in slightly higher location update cost on every agent migration than that of the previous forwarding pointer-based mechanism. However, if each agent chooses some among its visiting nodes as forwarders by properly considering several important performance factors, the gap between the two costs may be almost negligible.

\subsection{Data structures and algorithms}

\subsubsection{Directory service}

Every node $N_{i}$ should keep the following data structures in the directory service algorithm.

- MAExecuted ${ }_{i}$ : A table for saving location information of every agent currently running on $N_{i}$. Its element is a tuple ( $A I D, L I D, t s, m F l a g, m s g Q)$. LID is the identifier of agent $A I D$ 's locator. $t s$ is the timestamp associated with agent $A I D$ when the agent is located at $N_{i}$. Its value is incremented by one every time the corresponding agent migrates. Thus, when agent $A I D$ migrates to $N_{i}, N_{i}$ should inform $L I D$ of both its identifier and $t s$ so that $L I D$ can locate the agent. mFlag is a bit flag designating if the agent is currently migrating to another node $(=1) \operatorname{or} \operatorname{not}(=0) . m s g Q$ is a message queue for buffering all the messages destined to agent $A I D$ on its migration. Its element consists of two fields, a message $m$ and the source node ID of the message, $S I D$.

- FPointer $s_{i}$ : A table for saving location information of every mobile agent which is not currently running on $N_{i}$, but of which $N_{i}$ is a forwarder. Its element is a tuple (AID, NID, ts, lFlag, mFlag, 


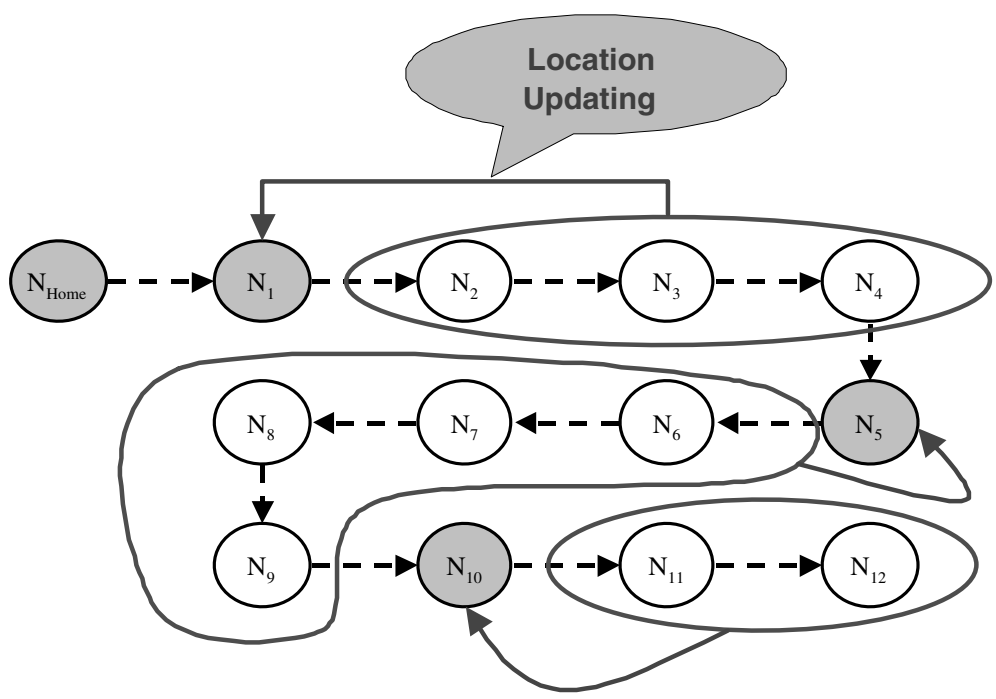

Fig. 4. Our scalable mechanism.

$m s g Q$ ). $N I D$ is the identifier of the node where $N_{i}$ knows agent $A I D$ is currently located and running. $t s$ is the timestamp associated with the agent when the agent is located at node $N_{N I D}$. It is used for avoiding updating recent location information by older information [16]. lFlag is a bit flag indicating whether $N_{i}$ is agent $A I D$ 's locator or not. In the first case, its value is 1 and otherwise, 0. mFlag is a bit flag designating if the agent is currently migrating to another node $(=1) \operatorname{or} \operatorname{not}(=0)$. Like in $M A$ Executed $_{i}, \mathrm{msg}_{\mathrm{Q}}$ is a message queue for buffering all the messages destined to agent $A I D$ on its migration. Its element consists of two fields, a message $m$ and the source node ID of the message SID.

The algorithm for managing each agent's location on its migration is informally described using Fig. 5. This figure shows message interactions between nodes occurring in $m a$ 's location updating while migrating from its home node to $N_{1}$ through $N_{5}$. In Fig. 5, $m a$ is created on $N_{\text {home }}$ and then an

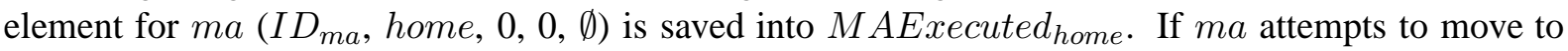
$N_{1}$ after having performed its partial task in the first step, it inserts into F Pointer shome ma's element (ID $D_{m a}, 1,1,1,1, \emptyset$ ) indicating $N_{\text {home }}$ is $m a$ 's locator and $m a$ is currently moving to $N_{1}$. Then, $N_{\text {home }}$ sends a message Migrated $(m a)$ to $N_{1}$ for dispatching the agent with the identifier of the first and $m a$ 's timestamp into the latter. When receiving these, $N_{1}$ increases the timestamp by one. In this case, as $m a$ wants $N_{1}$ to be its locator, it inserts ma's location information $\left(I D_{m a}, 1,1,1, \emptyset\right)$ into MAExecuted $d_{1}$. In the second step, $N_{1}$ sends $N_{\text {home }}$ a message Replaced $\left(I D_{m a}, 1\right)$ including ma's timestamp in order to inform $N_{\text {home }}$ that $N_{1}$ is $m a$ 's locator from now. On receiving the message, $N_{\text {home }}$ updates $m a$ 's location information in F Pointer shome using the message and sets two fields of ma's element, lFlag and mFlag, both to 0 . If the messages destined to $m a$ have been buffered in $N_{\text {home }}$ 's message queue due to the migration, they are transmitted to $N_{1}$. When $m a$ attempts to migrate to $N_{2}$ after $m a$ has performed a part of its task in the third step, $N_{1}$ puts $m a$ 's element $\left(I D_{m a}, 2,2,1,1, \emptyset\right)$ into F Pointer $s_{1}$ and then sends a message Migrated ( $\mathrm{ma}$ ) for moving agent $\mathrm{ma}$ to $\mathrm{N}_{2}$. In this case, $\mathrm{N}_{2}$ increments $\mathrm{ma}$ 's timestamp by one and then inserts $m a$ 's element $\left(I D_{m a}, 1,2,1, \emptyset\right)$ into MAExecuted $_{2}$ because $m a$ wants $N_{2}$ to be just a visiting node. In the fourth step, the node sends a message Register $\left(I D_{m a}, 2\right)$ including the timestamp to its locator $N_{1}$ for registering $m a$ 's current location with the locator. If there are any messages sent to the agent in the queue of $N_{1}$, they are forwarded to $N_{2}$. 


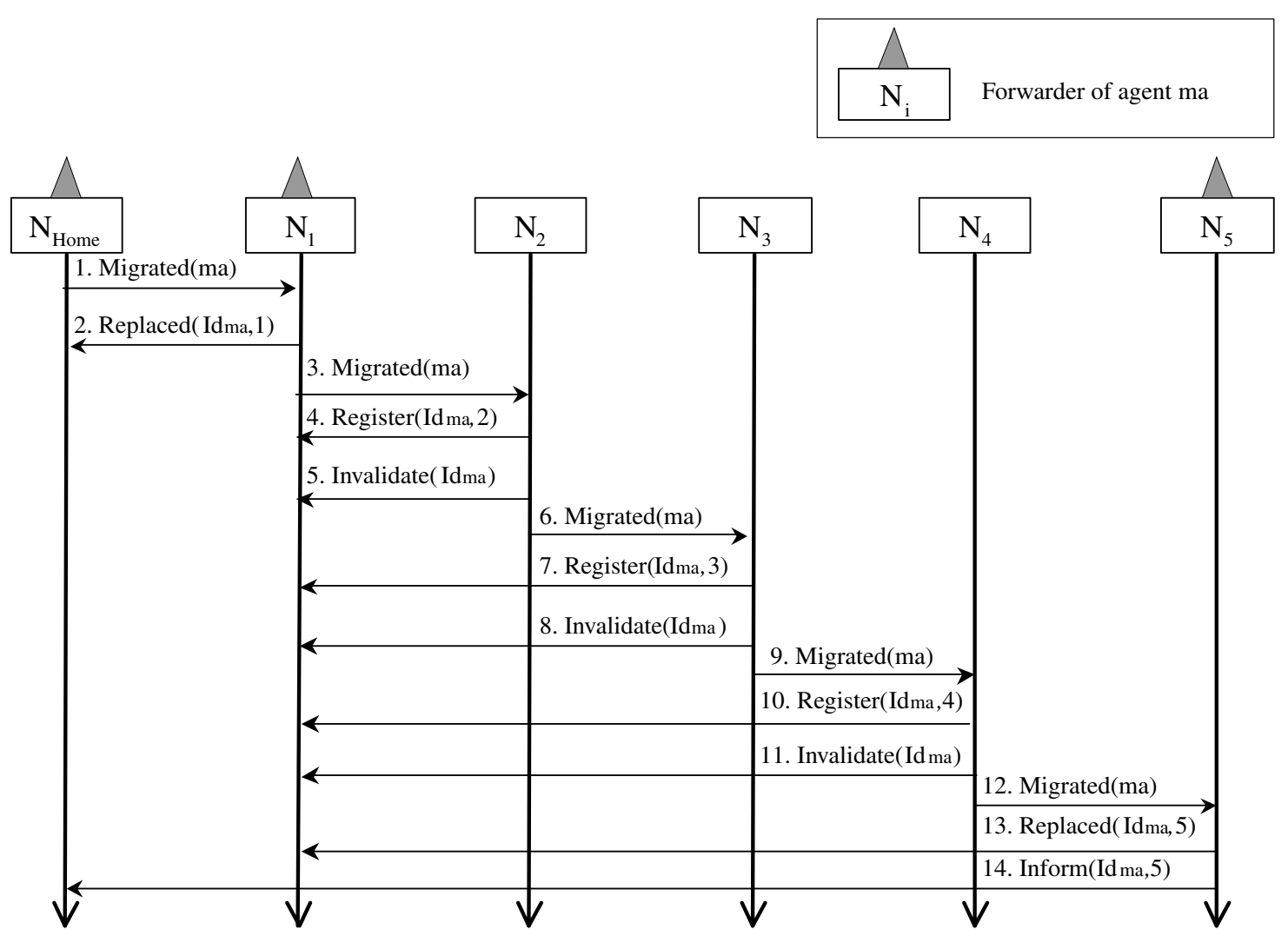

Fig. 5. An execution of our proposed directory service algorithm for an agent $\mathrm{ma}$ during its multi-node movement.

In the fifth step, $N_{2}$ first sends $N_{1}$ a message Invalidate $\left(I D_{m a}\right)$ indicating that ma's migration process begins from now. When receiving the message, $N_{1}$ sets one field of ma's element, mFlag, to 1. Suppose the migration is started without the execution of this invalidation procedure. If $N_{1}$ receives any message destined to $m a$ in this case, it forwards the message to $N_{2}$ because it doesn't know whether the migration continues to be executed. But, neither ma may be currently running on $N_{2}$ nor $N_{2}$ keep $m a$ 's location information on FPointer $s_{2}$ because $N_{2}$ isn't $m a$ 's forwarder. In this case, the message cannot be delivered to $\mathrm{ma}$. Therefore, $\mathrm{N}_{2}$ should transmit a message Migrated $(\mathrm{ma})$ to $\mathrm{N}_{3}$ after having performed the invalidation procedure on $m a$ 's locator, and then remove $m a$ 's element from $M$ AExecuted $d_{2}$. Afterwards, $m a$ 's visiting node $N_{3}$ increments $m a$ 's timestamp and saves $m a$ 's element $\left(I D_{m a}, 1,3,1, \emptyset\right)$ into $M A$ Executed $_{3}$ in the sixth step, and then sends a message Register $\left(I D_{m a}, 3\right)$ to $N_{1}$ in the seventh step. On the receipt of the message, $N_{1}$ updates ma's element in FPointer $s_{1}$ to $\left(I D_{m a}, 3,3,1,0, \emptyset\right)$ using the message. When $m a$ migrates to $N_{4}$, the same procedure is performed like in this figure. When agent $m a$ moves from $N_{4}$ to $N_{5}$ and decides that $N_{5}$ is the proper node as $m a$ 's locator, $N_{5}$ creates ma's location information $\left(I D_{m a}, 5,5,1, \emptyset\right)$ and inserts it into MAExecuted $d_{5}$ in the twelfth step. Afterwards, in the thirteenth step, the node sends $N_{1}$ a message Replaced $\left(I D_{m a}, 5\right)$ for notifying the previous locator $N_{1}$ that $N_{5}$ is $m a$ 's locator from now. Also, $m a$ may send a message In form $\left(I D_{m a}, 5\right)$ to $N_{\text {home }}$ for updating its current location at $N_{\text {home }}$ in the fourteenth step to reduce the message delivery time incurred when another agent initially sends a message to ma via $N_{\text {home }}$. If $m a$ recognizes this consideration helps no performance improvement, it doesn't perform the update procedure on the home node. 


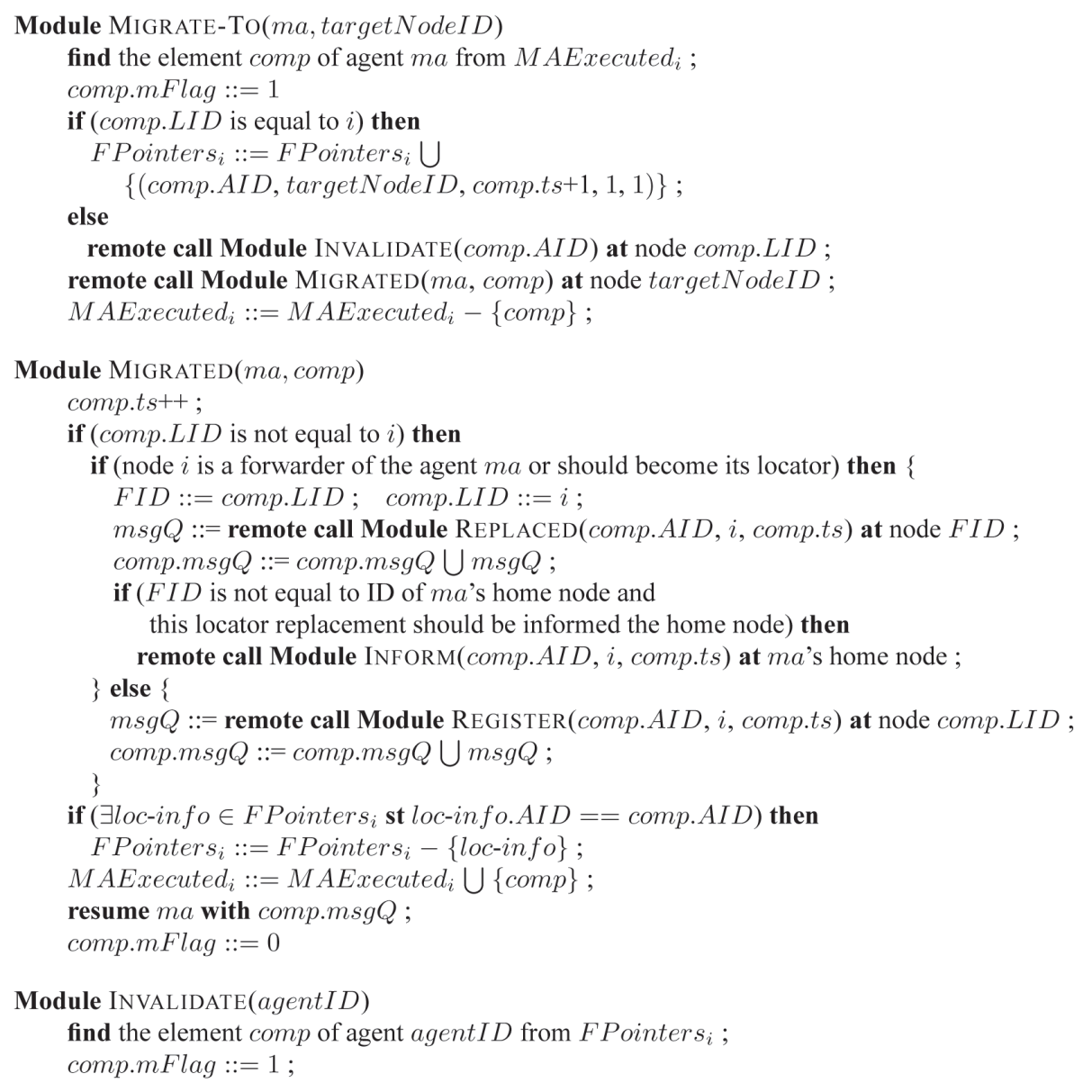

Fig. 6. Modules for a service node $i$ on agent migration.

The informally stated modules for every service node in the directory service algorithm are formally given in Figs 6 and 7.

\subsubsection{Message delivery}

Every node $N_{i}$ should have an agent location cache $M A L i n k s_{i}$ in the message delivery algorithm. It is a cache for temporarily storing location information of each mobile agent which agents running on $N_{i}$ communicate with. Its element is a tuple $(A I D, N I D, t s) . N I D$ is the identifier of the node where $N_{i}$ knows agent $A I D$ is currently located and running. Thus, when attempting to deliver messages to agent $A I D$, each agent on $N_{i}$ forwards them to NID regardless of whether this address is outdated. $t s$ is the timestamp assigned to agent $A I D$ when the agent was located at node $N_{N I D}$.

We intend to use an example in Fig. 8 to clarify the algorithm to enable every sent message to be reliably delivered to its target agent despite agent migrations. This example illustrates agent $\gamma$ sends three messages, $m_{1}, m_{2}$ and $m_{3}$ to agent $m a$ in this order while $m a$ is migrating from its home node to $N_{1}$ through $N_{5}$ according to its itinerary. In this figure, after $m a$ has moved from $N_{\text {home }}$ to $N_{3}, \gamma$ at $N_{w}$ attempts to deliver the first message $m_{1}$ to $m a$. In this case, $N_{w}$ has no location information for $m a$ in its location cache $M A L_{i n k} s_{w}$. Thus, $N_{w}$ creates and saves $m a$ 's element $\left(I D_{m a}\right.$, home, 0$)$ into 
Module Register(agentID, current NodeID, timestamp) find the element comp of agent agentID from FPointer $s_{i}$; comp.NID ::=currentNodeID; comp.ts ::=timestamp; comp.mFlag ::=0; return comp.msgQ ;

Module INFORM(agentID, locatorID, timestamp) find the element comp of agent agentID from FPointer $s_{i}$; comp.NID ::=locatorID ; comp.ts ::=timestamp;

Module REPLACED(agentID, locatorID, timestamp) find the element comp of agent agentID from FPointer $s_{i}$; comp.NID ::=locatorID; comp.ts ::= timestamp; comp.lFlag $::=0 ；$ comp.mFlag $::=0$; return comp.msgQ;

Fig. 7. Modules for a service node $i$ on agent migration (continued).

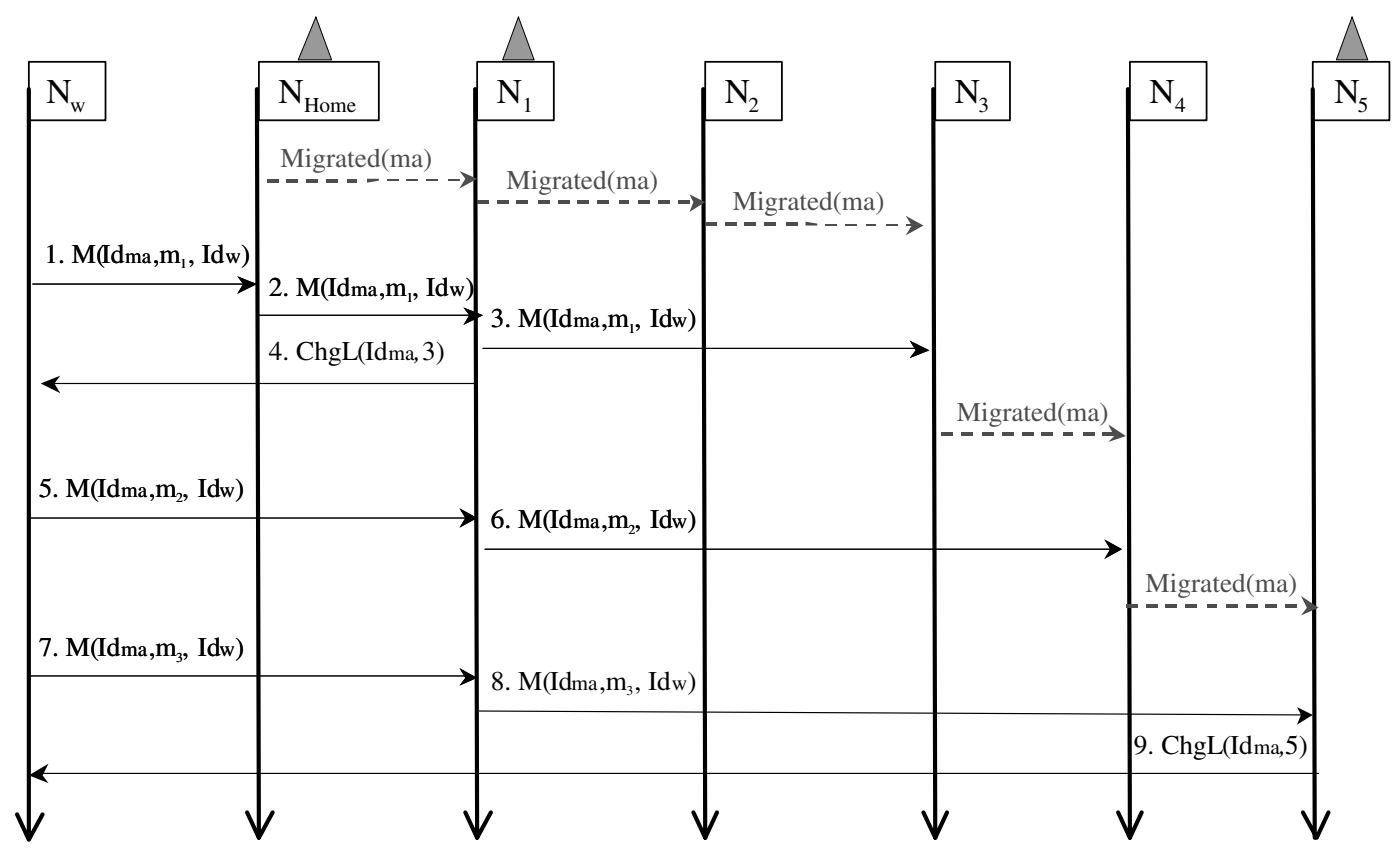

Fig. 8. An execution of reliably forwarding messages from their source agent $\gamma$ to their target agent ma during its multi-node movement.

$M A L i n k s_{w}$. Then, it sends the message $m_{1}$ to $N_{\text {home }}$. Receiving the message, $N_{\text {home }}$ retrieves $m a$ 's element from F Pointer shome. In this case, as the value of the bit flag lFlag in the element is $0, N_{\text {home }}$ isn't $m a$ 's locator. Thus, it consults the element and forwards the message $m_{1}$ to the next forwarder $N_{1}$. On the receipt of the message, $N_{1}$ obtains $m a$ 's element from F Pointer $s_{1}$ and then checks the flag lFlag in the element. In this case, $N_{1}$ is $m a$ 's locator because the value of the flag is 1 . Also, as the value of the second flag mFlag is 0 , it forwards the message to $m a$ 's currently running node $N_{3}$ by consulting the element. At the same time, as $N_{w}$ has the outdated identifier of $m a$ 's locator, $N_{1}$ sends $N_{w}$ a message $C h g L\left(I D_{m a}, 3\right)$ containing the identifier of $m a$ 's current locator $\left(=\mathrm{N}_{1}\right)$ and timestamp $(=3)$ in the figure. When receiving the message, $N_{w}$ updates ma's element in $M A L i n k s_{w}$ using the message. 


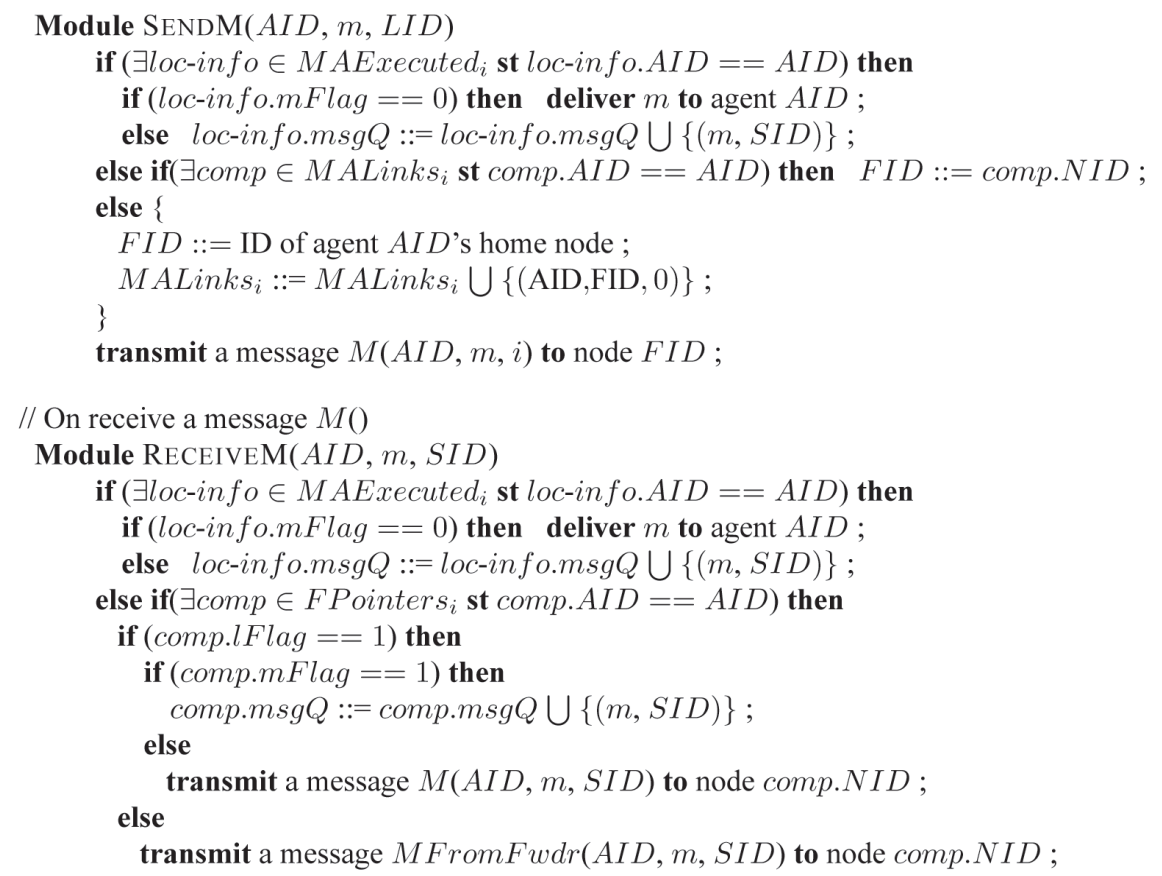

Fig. 9. Modules for a service node $i$ on message delivery.

When $m a$ migrates from $N_{3}$ to $N_{4}$ and then $\gamma$ at $N_{w}$ sends the second message $m_{2}$ to $m a, N_{w}$ finds $m a$ 's element from MALinks $s_{w}$ and then forwards $m_{2}$ to $N_{1}$. On the receipt of $m_{2}, N_{1}$ can see that it is $m a$ 's current locator because the value of the bit flag lFlag of $a$ 's element in F Pointer $s_{1}$ is 1 . Also, as the value of the flag $m$ Flag is $0, N_{1}$ can send $m_{2}$ to $m a$ 's currently running node $\left(=N_{4}\right)$. In this case, as $\gamma$ knows the identifier of $m a$ 's current locator, $N_{1}$ doesn't send any message $C h g L$ to $N_{w}$.

After $m a$ has migrated from $N_{4}$ to $N_{5}, \gamma$ transmits the third message $m_{3}$ to $m a$. At this point, $N_{w}$ sends $m_{3}$ to $N_{1}$ by consulting $m a$ 's element in MALinks $s_{w}$. In this case, as the value of the flag lFlag of ma's element in FPointer $s_{1}$ is $0, N_{1}$ forwards $m_{3}$ to the next forwarder $N_{5}$. When $N_{5}$ receives the message, it can deliver the message to $N_{5}$ because it recognizes that it is $m a$ 's locator and $m a$ is currently running on it. Concurrently, $N_{5}$ informs $N_{w}$ that $m a$ 's current locator is $N_{5}$ by sending a message $C h g L\left(I D_{m a}, 5\right)$ to $N_{w}$.

The modules for every service node in the message delivery algorithm are formally described in Figs 9 and 10.

\subsection{Correctness}

In this section, we prove the correctness of our proposed scalable communication mechanism.

Definition 1. ForwardingPath $\left(m s g, I D_{m a}, N_{i}\right)$ is a sequence $<N_{i}, \ldots, N_{n}>$ of a mobile agent $m a$ 's forwarders which compose an acyclic path for delivering a message $m s g$ from $N_{i}$ to $N_{n}$, the current locator of agent $m a$. For all $N_{j}, N_{k}$ in the path, if $i \leqslant j<k \leqslant n$, agent $m a$ visited $N_{j}$ and then $N_{k}$.

Theorem 1. When any message is sent to an agent in the system, the proposed mechanism enables the message to correctly be delivered to the target agent despite its migrations. 


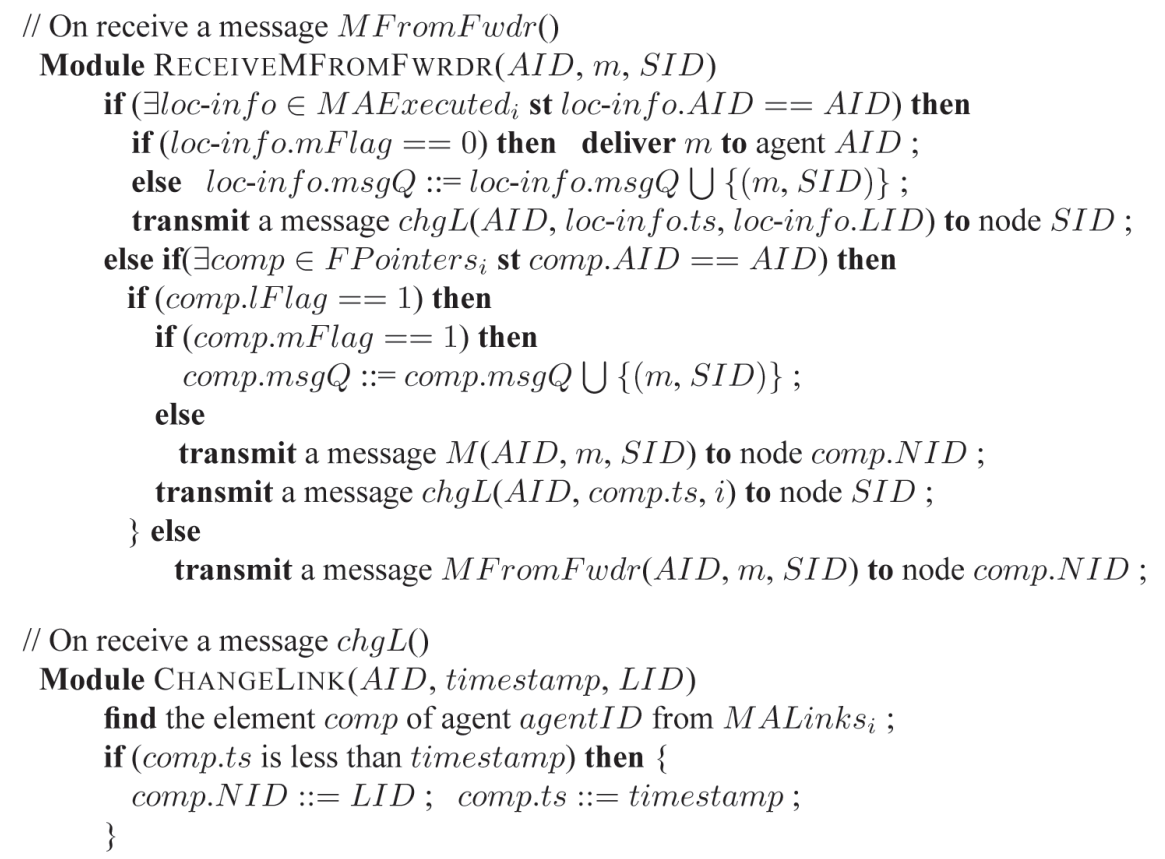

Fig. 10. Modules for a service node $i$ on message delivery (continued).

Proof. Assume that a message $m s g$ has to be delivered to its target agent $m a$. In this case, the sender agent of the message is $\gamma$ at node $N_{i}$ and agent $m a$ is currently running on node $N_{j}$. When $N_{i}$ attempts to send the message to agent $m a, N_{i}$ first looks up the address for $m a$ from its agent location cache MALinks $s_{i}$. If there is the address in the cache, $N_{i}$ sends the message to this address. Otherwise, it obtains the address of $m a$ 's home node from $m a$ 's identifier and then sends the message to the address. In both cases, suppose the forwarding address is denoted by $N_{l}$. The proof proceeds by induction on the number of all the forwarders $\in$ ForwardingPath $\left(m s g, I D_{m a}, N_{l}\right)$, denoted by |ForwardingPath $\left(m s g, I D_{m a}, N_{l}\right) \mid$.

\section{[Base case]}

In this case, $N_{l}$ is the locator of agent $m a$. Therefore, the following two cases should be considered.

Case 1: $l=j$.

In this case, $N_{j}$ can find $m a$ 's location information $e$ from $M A E x e c u t e d_{j}$. There are two subcases considered.

Case 1.1: e.mFlag $=0$.

In this case, the message $m s g$ is trivially delivered to $m a$ because $m a$ is currently running on node $N_{j}$. Case 1.2: e.mFlag $=1$.

In this case, agent $m a$ attempts to move to another node $N_{j+1}$. Thus, the message is saved on $m a$ 's message queue e.msgQ in $M A E x e c u t e d_{j}$ until the movement process is completed. After agent $m a$ with the element $e$ including the message has been migrated to $N_{j+1}$, the message can be correctly delivered to $m a$ when the agent resumes its execution on the node $N_{j+1}$.

Case 2: $l<j$.

In this case, $N_{l}$ looks up $m a$ 's location information $e$ from FPointer $s_{l}$. Then, it checks the value of a flag variable e.mFlag. There are two subcases according to the value.

Case 2.1: e.mFlag $=0$. 
In this case, the message is sent directly to $N_{j}$. Then, the subsequent procedure is performed like in case 1. Therefore, $m a$ can correctly receive the message.

Case 2.2: e.mFlag $=1$.

In this case, agent $m a$ is migrating to another node $N_{j+1}$. Thus, the message is buffered into $m a$ 's message queue e.msgQ in FPointer $s_{l}$ until the movement process is completed. After the migration, the value of a flag variable e.mFlag changes to 0 and then the message recorded in the queue is forwarded to $N_{j+1}$. Therefore, the message can be correctly delivered to $m a$.

\section{[Induction hypothesis]}

We assume that the theorem is true for the message msg in case that |ForwardingPath $\left(m s g, I D_{m a}, N_{l}\right) \mid=k$.

\section{[Induction step]}

After the message has been safely routed to the $k$-th forwarder $N_{\alpha}$ by induction hypothesis, $N_{\alpha}$ retrieves $m a$ 's location information $e$ from $F$ Pointer $s_{\alpha}$. Then, it forwards the message to the $(k+1)$-th forwarder $N_{\beta}$, which is the locator of agent $m a$. The following case is similar to the base case mentioned above. Therefore, the message can be correctly delivered to $m a$.

By induction, the mechanism can correctly deliver any sent message $m s g$ to its corresponding agent even if the agent migrates.

\section{Experimental evaluation}

In this section, we compare performance of our proposed mechanism(Proposed) with that of an existing forwarding pointer-based one(Existing) [16] by performing an extensive simulation using a discrete-event simulation language [2]. There are two performance indices as follows. The first is $\mathrm{Hop}_{\mathrm{Cntr}}$, the average number of nodes which have forwarded a message destined to a mobile agent until the message is actually delivered to the agent. The second is $L I n f o_{n o}$, the average number of agent location entries that each service node keeps on its storage.

A simulated system is composed of 100 nodes each associated with a coordinate(x,y). They are all interconnected with each other. Any two adjacent nodes are connected with a LAN link having a bandwidth of $10 \mathrm{Mbps}$ and a propagation delay of $2 \mathrm{~ms}$. For simplicity of this simulation, we assume that both bandwidth and propagation delay between any pair of nodes are proportional to their distance. In our simulation environment, there are some important simulation parameters as follows. The first is $S_{\text {snd }}$, the set of nodes being capable of sending messages to a mobile agent. Nodes in the set are uniformly distributed along the entire network. The second is $L C h g_{t h r d}$, the threshold which is required when a mobile agent decides whether the current locator of the agent should be changed. If a randomly generated probability variable $\delta$ for an agent $m a$ is greater than its threshold $L C h g_{t h r d}$ when the agent attempts to migrate to another node, the next node becomes the locator of the agent. In this simulation, it is assumed that the threshold $L C h g_{t h r d}$ is determined when its agent is created and isn't changed during the entire life cycle of its agent any more. The third is Stay $_{\text {time }}$, the mean time elapsed when a mobile agent stays at a node for performing its task, following an exponential distribution. The last simulation

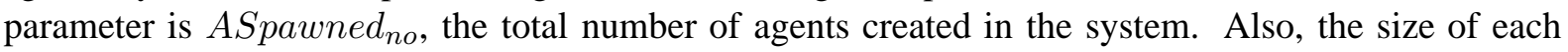
application message transmitted between any two agents ranges from 512 bytes to 1 Kbytes and the size of each control message for the inter-agent communication is 128bytes. All experimental results shown in this simulation are all averages over a number of trials.

Figure 11 shows the average message forwarding overhead, $\mathrm{Hop}_{\mathrm{Cntr}}$, for the two directory service and message delivery mechanisms for the specified range of the Staytime. In this figure, as the Staytime of 


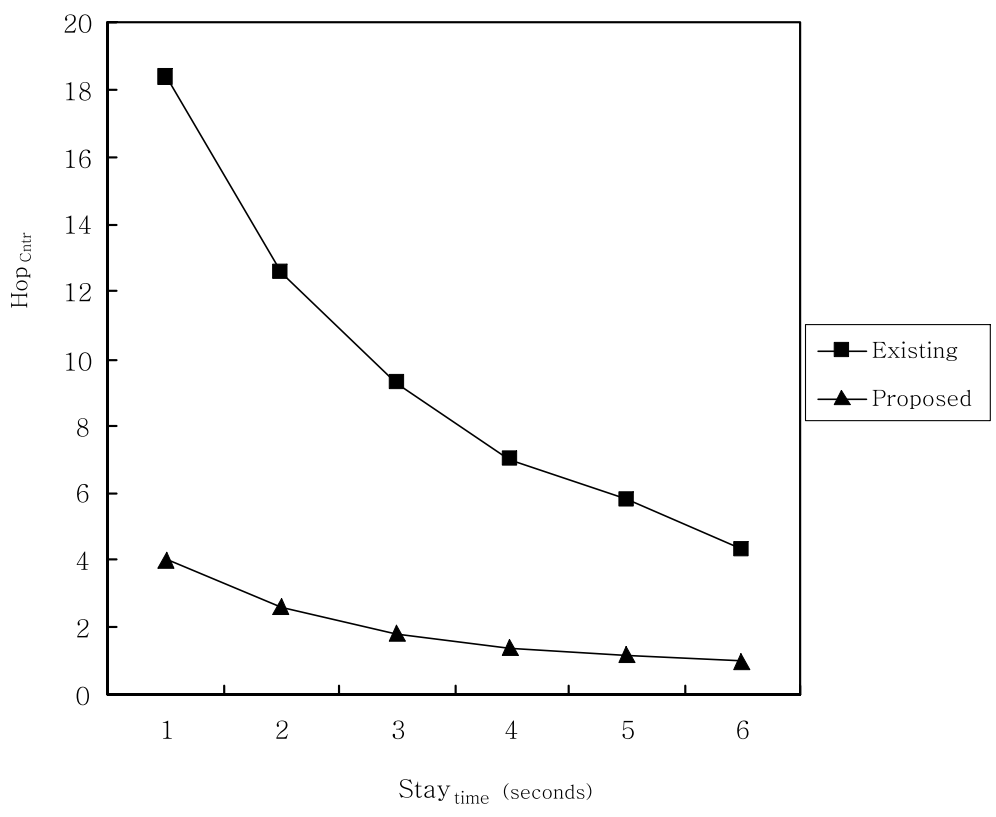

Fig. 11. Hop Cntr $_{\text {vs. Staytime. }}$

the two mechanisms decreases, their $H_{o p} p_{n t r}$ s increase. The reason is that as the length of each agent's stay grows shorter, the path for forwarding messages to the agent is contrarily made longer. However, we can see that the Hop Cntr of Proposed is significantly lower than that of Existing in this figure. In particular, as the Staytime becomes shorter, the gap between their message delivery costs considerably grows larger. In these results, the mechanism Proposed reduces about 76-79\% of $\mathrm{Hop}_{\mathrm{Cntr}}$ compared with the mechanism Existing.

Figure 12 shows the amount of agent location information kept by each service node, $L$ In $o_{n o}$, for the two mechanisms with varying the number of agents generated in the simulated system, ASpawned ${ }_{n o}$. As shown in this figure, increasing the ASpawned $_{n o}$ of the two mechanisms causes their Info $_{n o}$ s to grow higher accordingly. This is because as the number of agents created in the system increases, the two mechanisms are stepping up the amount of agent location information maintained by each node. But, as expected, Proposed provides very high performance gains in LInfo ${ }_{n o}$ over Existing. Especially, when ASpawned $_{n o}$ is increasing, the increasing rate of $L I n$ fo $_{n o}$ of Proposed is rising much slower than that of Existing. In this simulation, the mechanism Proposed reduces $L I n f o_{n o}$ by $79 \%$ compared with the mechanism Existing.

Therefore, these simulation results show that even if the scale of a mobile agent system grows considerably, our proposed mechanism Proposed incurs low message forwarding overhead and reduces the delivery time of each message destined to a mobile agent and the amount of agent location information kept by each service node compared with the previous one Existing.

\section{Related work}

In Mole [4], it is assumed that an agent never migrates while communicating with another. Thus, if corresponding agents move, their communication is implicitly terminated. It maintains a trail of pointers 


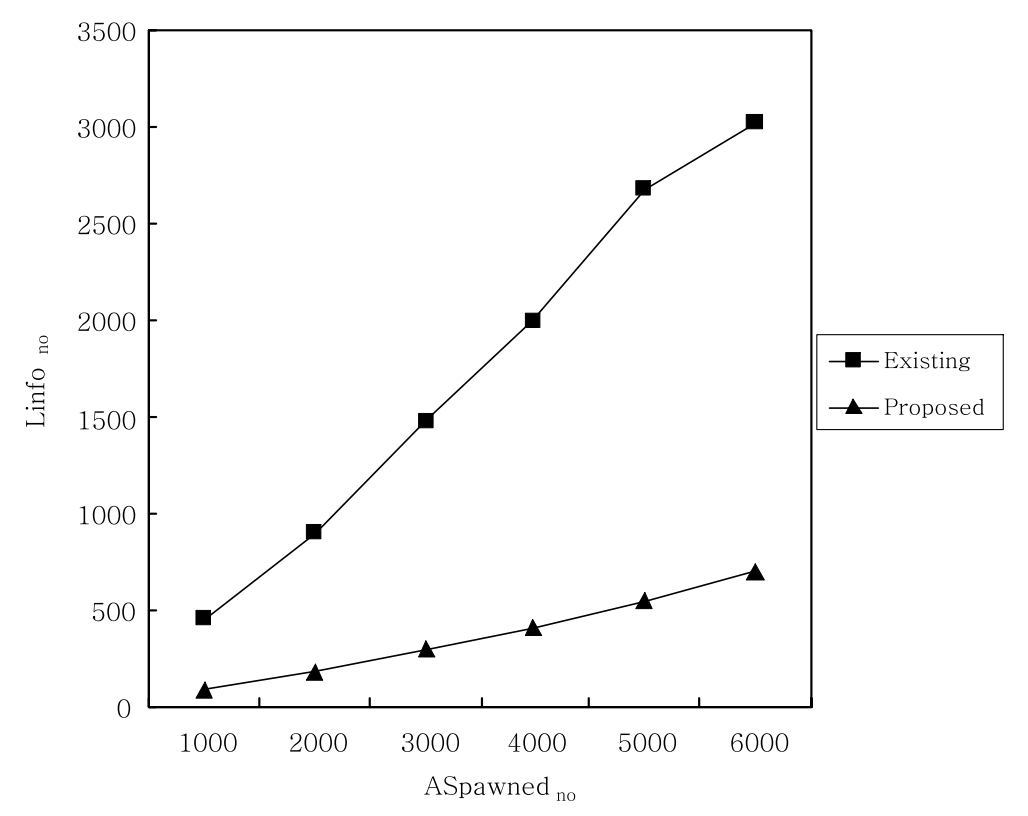

Fig. 12. Infono $_{\text {vs. ASpawned }}$.

of each agent for faster communication. But, this is exploited only in the context of a orphan detection protocol. Also, Mole supports group communication for a set of agents being non-mobile during a set of information exchanges.

A broadcast-based mobile agent communication mechanism was proposed by Murphy and Picco [18]. The mechanism guarantees transparent and reliable inter-agent communication and can also provide multicast communication for a set of agents. But, to locate the message destination, it has to contact every visiting host in the network. Thus, its large traffic overhead makes broadcasts impractical in large-scale mobile agent systems.

Concordia [27] supports inter-agent communication implemented via events. Each agent cannot raise and listen to events until it should register with an event manager. Subsequently, the sender agent connects with the event manager and posts an event with its request. As soon as the event manager receives a particular event, it informs all registered agents of the event. Although an agent migrates to another node, it can still receive requests from the event manager. It can register with any number of event managers. But, for one agent to communicate with another agent, the recipient agent should know the event manager location exactly.

Belle [6] proposed a hierarchical structure-based mechanism to form a location directory consisting of a hierarchy of servers. The location server at each level keeps lower-level object location information. For each object, the information is either a pointer to an entry at a lower-level location server or the agent's actual current location. However, this hierarchy cannot always be easily formed, especially in the Internet environment. Moreover, this mechanism may cause useless hops to be taken along the hierarchy.

In [24], a resending-based message delivery mechanism was introduced to use TCP-like slidingwindow protocols for detecting the loss of a message and retransmitting it. After several resendings, the sender contacts the location server and transmits the message to the new destination. But, the mechanism needs synchronizing the message routing with agent migration.

A distributed location directory management mechanism for mobile hosts [23] was proposed to be able to adapt to changes in geographical distribution of mobile hosts population in the network and to changes 
in mobile host location query rate. The mechanism replicates location information about mobile hosts at $O(\sqrt{m})$ ( $m$ is the total number of base stations in the system). This mechanism allows frequently queried mobile hosts to have their location information saved on a greater number of base stations. Thus, it may improve lookup and update performance by using replication. However, it is unsuitable for mobile agent communication because the highly dynamic nature and extremely fast migration speed of software mobile agents unlike mobile hosts make it very difficult to get the information about the distribution patterns and location query rates of a large number of mobile agents immediately, which may result in enormously high communication overhead.

Moreau [16] proposed a distributed directory service and message routing mechanism based on forwarding pointers for mobile agents. In this mechanism, each mobile agent is associated with a timestamp that is increased every time the agent migrates. This timestamp avoids cyclic routing when the agent migrates to previously visited hosts. Also, this mechanism introduces a type of message in form, containing an agent's location and the timestamp it had at that location, in order to reduce chains of forwarding pointers. A host that receives an inform message is allowed to update its table if the received information is more recent than the one it had. However, it specifies no concrete and efficient solutions for this purpose, for example, when inform messages must be sent, and which host they should be sent to.

Feng [8] introduced a mailbox-based mechanism to provide location-independent reliable message delivery. It allows messages to be forwarded at most once before they are delivered to their receiving agents. Also, the movement of agents can be separated from that of their mailboxes by determining autonomously whether each mailbox is migrated to its owner agent. However, uncertainty of message delivery to mailboxes may result in useless early pollings. On the other hand, even if urgent messages are forwarded to a mailbox on time, they can be delivered to its corresponding agent very late depending on the agent's polling time. Moreover, whenever each mailbox moves, its new location information should be broadcasted to every node where the mailbox has visited. This may incur high traffic overhead if assuming most agents are highly mobile.

Coordination models offer a more asynchronous form of communication, typically involving a tuple space [7]. As coordination spaces are non-mobile, they may result in the centralization problem like the home agent based mechanism. To alleviate this problem, the tuple space can be implemented in a distributed manner. But, consistency maintenance overhead is not negligible. Moreover, in this approach, coordinated processes have to poll tuple spaces, which leads to some difficulties with respect to both communication and computation. As a result, tuple spaces generally provide a mechanism by which registered clients can be informed of the arrival of a new tuple. If clients are mobile, the problem of how to deliver such notifications correctly occurs. When the tuple space is mobile [20], message delivery to the space should be also considered.

\section{Conclusion}

This paper introduced a scalable distributed directory and message delivery mechanism for mobile agents to require a small size of storage of each service node for agent location management and incur low message forwarding overhead while reducing the dependency on the home node. To achieve this goal, this mechanism forces only a small number of nodes among its visiting ones of each agent to become forwarders. It has each agent register its location with its current locator on every migration until it arrives at the next locator of the agent. This behavior may result in slightly higher location update cost per agent migration compared with that of the previous forwarding pointer-based mechanism. However, 
if each agent determines some among its visiting nodes as forwarders by properly considering several performance factors, the gap between the two costs may be almost negligible. Moreover, its message delivery algorithm enables each sending agent to communicate with mobile agents very fast by effectively managing and using their bindings in its agent location cache. Our experimental results demonstrated that the proposed mechanism considerably reduces both the amount of agent location information maintained by each service node and the delivery time of each message destined to a mobile agent compared with the existing one.

For future work, our research group are currently developing some methods for selecting appropriate forwarders of each mobile agent according to the changes which the agent senses in its environment related to location updating and message delivery costs, security policies, network latency and topology, communication patterns. In addition, we attempt to extend our mechanism to address fault-tolerance of forwarders of each mobile agent by using some effective information redundancy technique while reasonably preserving scalability of our mechanism.

\section{References}

[1] K. Alexandros and S. Joseph, A Trustworthy Mobile Agent Infrastructure for Network Management, In Proc. of the 10th IFIP/IEEE International Symposium on Integrated Network Management (2007), 383-390.

[2] R. Bagrodia, R. Meyer, M. Takai, Y. Chen, X. Zeng, J. Martin and H. Y. Song, Parsec: A Parallel Simulation Environments for Complex Systems, IEEE Computer (1998), 77-85.

[3] F. Baschieri, P. Bellavista and A. Corradi, Mobile Agents for Qos Tailoring, Control and Adaptation over the Internet: The UbiQoS Video on Demand Serbvice, In Proc. of the 2nd International Symposium on Applications and the Internet (2002), 109-118.

[4] J. Baumann, F. Hohl, K. Rothermel and M. Strasser, Mole-Concepts of a Mobile Agent System, World Wide Web 1(3) (1998), 123-137.

[5] P. Bellavista, A. Corradi and C. Stefanelli, The Ubiquitous Provisioning of Internet Services to Portable Devices, IEEE Pervasive Computing 1(3) (2002), 81-87.

[6] W. Belle, K. Verelst and T. D'Hondt, Location transparent routing in mobile agent systems merging name lookups with routing, In Proc. of the 7th IEEE Workshop on Future Trends of Distributed Computing Systems (1999), 207-212.

[7] G. Cabri, L. Leonardi and F. Zambonelli, Reactive Tuple Spaces for Mobile Agent Coordination, In Proc. of the 2nd International Workshop on Mobile Agents, Lecture Notes In Computer Science 1447 (1998), 237-248.

[8] J. Cao, X. Feng, J. Lu and S. Das, Mailbox-based scheme for mobile agent communications, IEEE Computer 35(9) (2002), 54-60.

[9] J. Cui and H. Chae, Mobile Agent based Load Balancing for RFID Middlewares, In Proc. of International Conference on Advanced Computer Technology (2007), 973-978.

[10] C. Curino, M. Giani, M. Giorgetta, A. Giusti, A. Murphy and G. Picco, Mobile Data Collection in Sensor Networks: The TinyLime Middleware, Journal of Pervasive and Mobile Computing 4(1) (2005), 446-469.

[11] J. Desbiens, M. Lavoie and F. Renaud, Communication and tracking infrastructure of a mobile agent system, In Proc. of the 31st Hawaii International Conference on System Sciences 7 (1998), 54-63.

[12] A. Fuggetta, G.P. Picco and G. Vigna, Understanding Code Mobility, IEEE Transactions on Software Engineering 24(5) (1998), 342-361.

[13] M. Fukuda, K. Kashiwagi and S. Kobayashi, AgentTeamwork: Coordinating Grid-Computing Jobs with Mobile Agents, In Special Issue on Agent-Based Grid Computing, International Journal of Applied Intelligence (2006).

[14] D. Lange and M. Oshima, Programming and Deploying Mobile Agents with Aglets, Addison-Wesley (1998).

[15] Z. Li and M. Parashar, A Decentralized Agent Framework for Dynamic Composition and Coordination for Autonomic Applications, In Proc. of the 3rd International Workshop on Self-Adaptive and Autonomic Computing Systems, Copenhagen, Denmark (2005), 165-169.

[16] L. Moreau, Distributed Directory Service and Message Router for Mobile Agents, Science of Computer Programming 39(2-3) (2001), 249-272.

[17] L. Moreau and D. Ribbens, Mobile Objects in Java, Scientific Programming 10(1) (2002), 91-100.

[18] A.L. Murphy and G.P. Picco, Reliable Communication for Highly Mobile Agents, Journal of Autonomous Agents and Multi-Agent Systems 5(1) (2002), 81-100.

[19] A.L. Murphy and G.P. Picco, Using Lime to Support Replication for Availability in Mobile Ad Hoc Networks, In Proc. of the 8th International Conference on Coordination Models and Languages (2006), 194-211. 
[20] A.L. Murphy, G.P. Picco and G.-C. Roman, Lime: A Coordination Middleware Supporting Mobility of Hosts and Agents, ACM Transactions on Software Engineering and Methodology 15(3) (2006), 279-328.

[21] C. Perkins, IP Mobility Support, RFC 2002 (1996).

[22] V. Pham and A. Karmouch, Mobile Software Agents: An Overview, IEEE Communications Magazine 36 (1998), $26-37$.

[23] R. Prakash and M. Singhal, A Dynamic Approach to Location Management in Mobile Computing Systems, In Proc. of the International Conference on Software Engineering and Knowledge Engineering (1996), 488-495.

[24] M. Ranganathan, M. Bednarek and D. Montgomery, A Reliable Message Delivery Protocol for Mobile Agents, In Proc. of the 2nd International Symposium on Agent Systems and Applications and the 4th International Symposium on Mobile Agents, Lecture Notes In Computer Science 1882 (2000), 206-220.

[25] K. Rothermel and M. Schwehm, Mobile Agents, Encyclopedia for Computer Science and Technology 40 (1999), 155-176.

[26] R. Tahboub and V. Lazarescu, Novel Approach for Remote Energy Meter Reading Using Mobile Agents, In Proc. of the 3rd International Conference on Information Technology (2006), 84-89.

[27] D. Wong, N. Paciorek, T. Walsh, J. DiCelie, M. Young and B. Peet, Concordia: An Infrastructure for Collaborating Mobile Agents, Lecture Notes In Computer Science 1219 (1997).

[28] J. Zhang, A mobile agents-based approach to test the reliability of web services, International Journal of Web and Grid Services 2(1) (2006), 92-117.

Jinho Ahn received his B.S., M.S. and Ph.D. degrees in Computer Science and Engineering from Korea University, Korea, in 1997, 1999 and 2003, respectively. He has been an assistant professor in Department of Computer Science, Kyonggi University. His research interests include distributed computing, fault-tolerance, sensor networks and mobile agent systems. He has published more than 50 papers in refereed journals and conference proceedings and served as program committee member or session chair in several domestic/international conferences. 

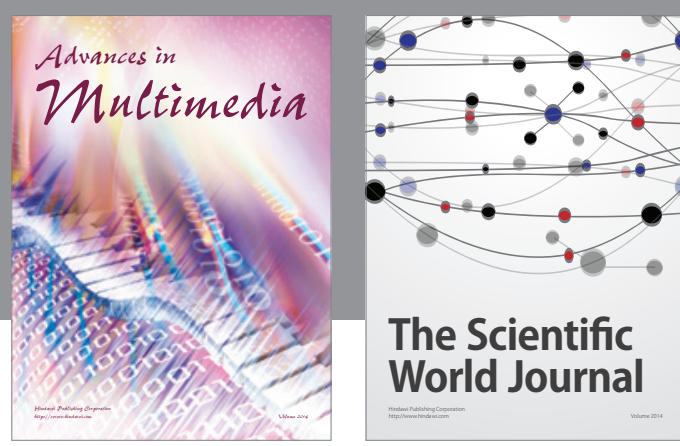

The Scientific World Journal
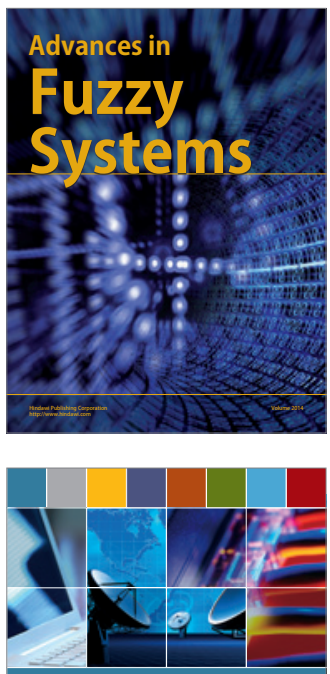

Computer Networks and Communications
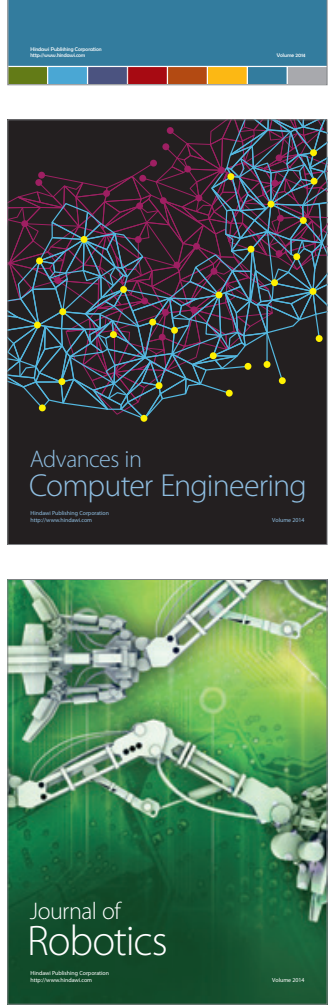
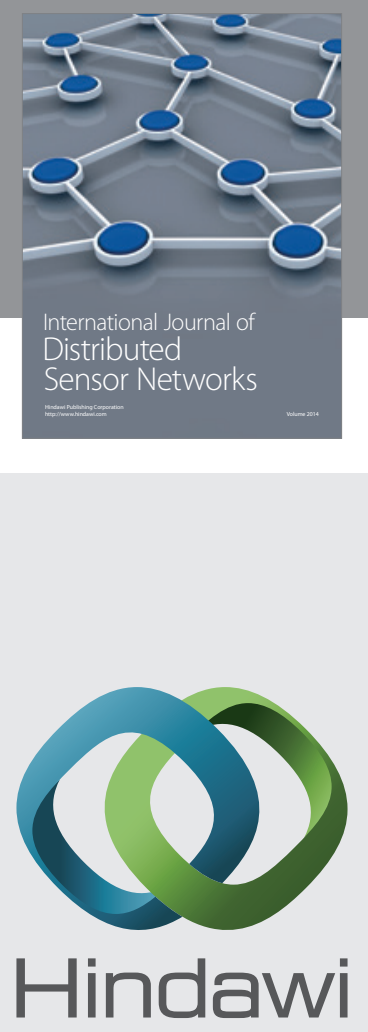

Submit your manuscripts at

http://www.hindawi.com
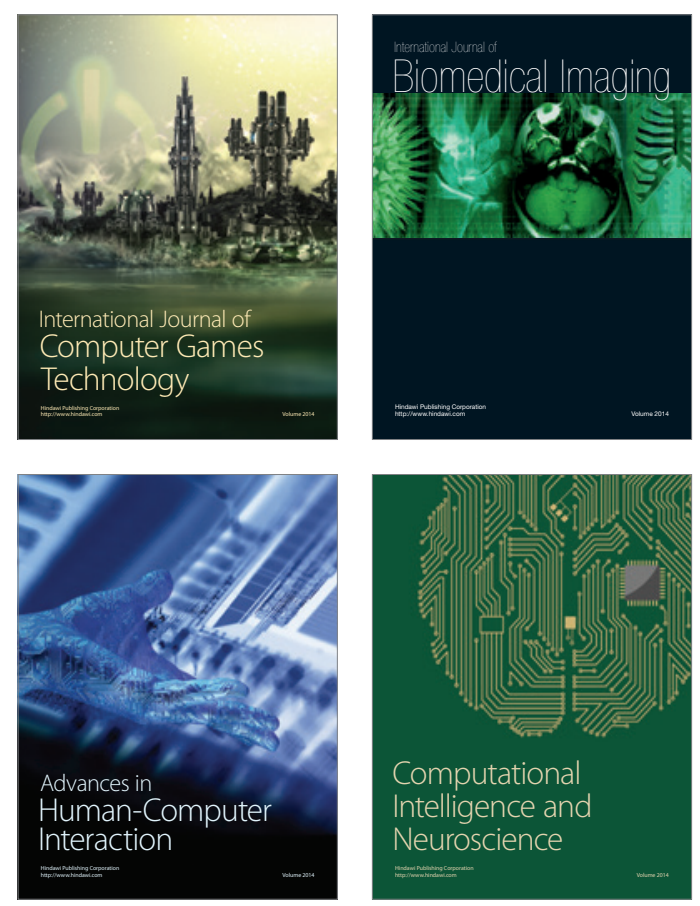
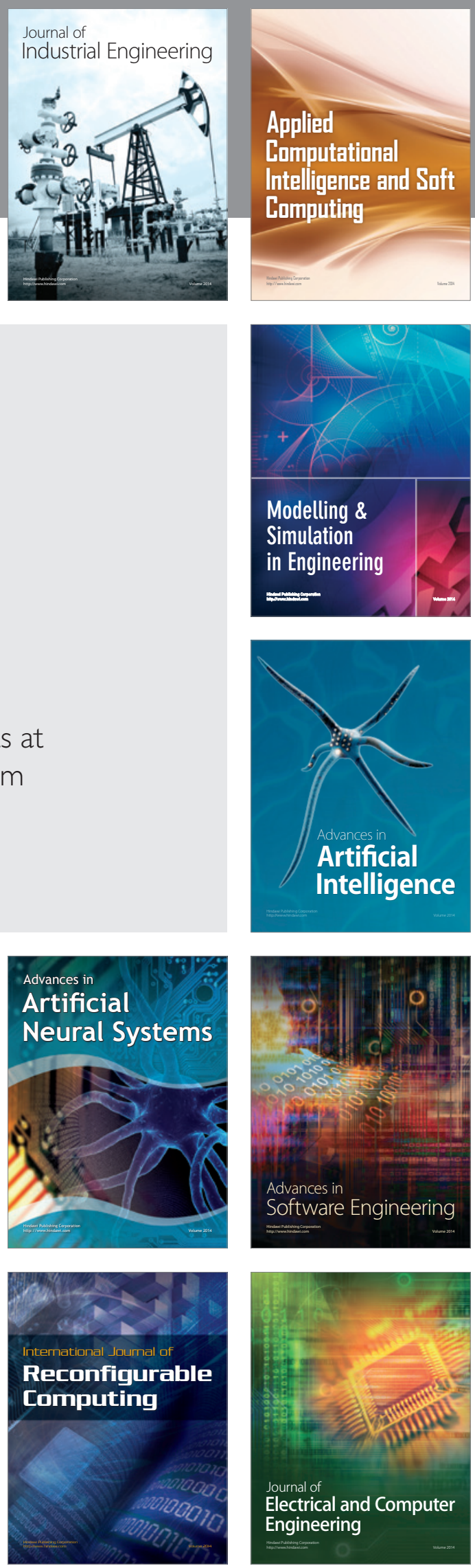